\title{
IMAGEN DE LA INMIGRACIÓN A TRAVÉS DE LAS PUBLICACIONES CIENTÍFICAS
}

\author{
Susana Limia Redondo* \\ Paloma Salvadores Fuentes** \\ * Profesora Colaboradora de Fundamentos de Enfermería \\ **Profesora. Catedrática de Fundamentos de Enfermería, \\ Administración de los Servicios de Enfermería y Documentación Sanitaria en la \\ Universidad Rey Juan Carlos
}

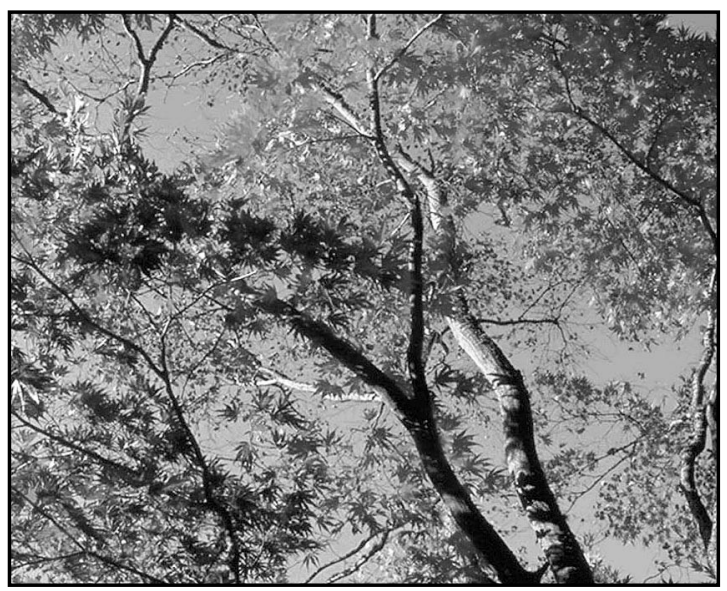

IMAGE OF IMMIGRATION THROUGH SCIENTIFIC LITERATURE

\section{ABSTRACT \\ Objective:}

To know the image the scientific community has about the health of immigrants, searching Spanish bibliography through the main Health Sciences databases

\section{Materials and Methods:}

In a first phase of the study we performed a bibliometric analysis encompassing the period 1980-2001. The searched databases were Medline and Indice Médico Español (IME). A second phase consisted in locating primary sources and analyzing the dominant discourse in each of the retrieved articles.

\section{Results:}

The search resulted in the identification of 80 articles complying with the search criteria (72 from Medline and 8 from IME). Regarding the bibliometric study, the Spanish autonomous region where most studies have been carried out was Catalonia (42.1\%), closely followed by Madrid (32.57\%).

Immigrants came from Africa (57\%), Latinamerica (30\%) and Asia (13\%)

The analysis of the dominant discourse within the articles can be summarized in the following two headings:

"Immigration results in an increase of infectious and tropical diseases".

"The use of health care services by the immigrants":

\section{Conclusions:}

There is a clear need for the implementation of interdisciplinary sociosanitary programs which may help to overcome language and cultural barriers. Different lifestyles, and hygienic, sanitary and alimentary practices and not the immigrant himself, represent a hazard for both individuals and public health.

Key words: health, immigration, bibliometrics, cualitative analysis, nur

\section{RESUMEN \\ Objetivo:}

Conocer la imagen que tiene la comunidad científica sobre la salud de los inmigrantes a través de la bibliografía española en las principales bases documentales de Ciencias de la Salud .

Material y métodos:

En la primera fase del estudio se ha realizado 
un análisis bibliométrico, que abarca el periodo 1980 -2001. Las bases de datos consultadas han sido Medline e Indice Médico Español (IME). La segunda fase del estudio, consistió en la localización de las fuentes primarias y su posterior análisis del discurso dominante en cada uno de los artículos recuperados.

\section{Resultados:}

Se registraron un total de 80 publicaciones que cumplieran los criterios marcados de la búsqueda bibliográfica de los cuales 72 publicaciones se encuentran indizadas en la base de datos Medline, y los 8 restantes pertenecen a la base de datos IME.

Respecto al análisis bibliométrico podemos afirmar que las comunidades españolas en las cuales se ha realizado el mayor número de estudios sobre inmigración y salud han sido Cataluña con un $42,1 \%$, seguida muy de cerca por la Comunidad de Madrid con total de 31,57\% estudios realizados sobre inmigración.

En cuanto a la procedencia de los inmigrantes que formaban parte de los artículos estudiados fue la siguiente: $57 \%$ África, 30\% Latinoamérica y $13 \%$ Asia.

Referente al análisis de discurso, podemos decir que existen dos discursos dominantes, que se muestran en los siguientes epígrafes:

"La inmigración produce un aumento de las tasas de Enfermedades Infecciosas y Tropicales"

"El uso de los servicios de salud por la población inmigrante".

\section{Conclusiones:}

Existe una clara necesidad para la puesta en marcha de programas interdisciplinarios sociosanitarios que ayuden a superar las barreras culturales e idiomáticas.

El inmigrante en sí no constituye un riesgo para la salud pública, sino que son los estilos de vida, las prácticas higiénicas, sanitarias y alimenticias, las que representan un riesgo para la salud pública e individual.

Palabras clave: salud, inmigración, bibliometria, análisis discurso, enfermería.

\section{INTRODUCCIÓN}

Actualmente, estamos viviendo en la época de la "medicina socializada" o de una medicina comunitaria, en la cual se revaloriza el papel de la población en el campo sanitario. Además hay que considerar la salud como un bien comunitario, y como tal, implica la colaboración de todos los ciudadanos, tanto a nivel colectivo como individual, en pro de su consecución. Este nuevo enfoque, nos lleva a considerar la salud, no sólo como la ausencia de enfermedad, sino un estado de bienestar físico, psíquico y social (Carta Magna 1946).

Esta tendencia queda reflejada en la propuesta de la Organización Mundial de la Salud en la reunión de Alma-Ata (WHO 1979) , titulada; "un aceptable nivel de salud para toda la población del mundo en el año 2000" , viene remarcada por distintos artículos de la misma declaración que pone énfasis en la participación (como derecho y obligación) de la población (a nivel comunitario e individual) en las tareas relacionadas con su atención sanitaria. Con este cambio, la población deja de ser un mero receptor de una medicina en la cual el médico era dueño y señor, para pasar a ser parte integrante fundamental de los procesos que tienen como meta una mejora de su nivel de salud, dentro de un trabajo en equipo del que el paciente es ya un miembro del equipo. Se entiende, por lo tanto una participación de la población en los asuntos sanitarios como forma de superar los problemas de deshumanización que la tradicional asistencia comportaba, y resolver, o suavizar al menos, las desigualdades de origen socio-económico que la institución sanitaria ha reproducido y mantenido hasta la fecha.

La OMS insiste cada vez más en que la población, como paciente potencial que es, debe participar en la planificación de la política sanitaria, en su ejecución y en la evaluación del sistema sanitario.

Por otra parte, el derecho a la salud a nivel Internacional, queda recogido por primera vez en 1948 en el artículo 25 de la Declaración Universal de Derechos Humanos, que afirma; " Toda persona tiene derecho a un nivel de vida adecuado, que le asegure, así como a su familia, la salud y el bienestar, la asistencia médica y los servicios sociales necesarios" 
Finamente, debido a este ímpetu en el concepto de medicina socializada, de lo colectivo, de la participación comunitaria, y teniendo en cuenta el incremento que se está produciendo en España de la población inmigrante, nos hizo cuestionarnos si en la comunidad científico-sanitaria se estaban registrando o escribiendo estos acontecimientos, y si era así, qué documentación se estaba recopilando o creando, en definitiva, qué marco referencial se está construyendo respecto a la salud de los inmigrantes.

\section{MATERIAL Y MÉTODO}

El objetivo principal del estudio es: Conocer la imagen que tienen la comunidad científico-sanitaria de la salud de los inmigrantes, a través bibliografía española existente de las principales bases documentales en Ciencias de la Salud .

Los objetivos complementarios que nos hemos propuestos en una segunda etapa, siempre que se recupere un o se obtenga un volumen bibliográfico pertinente, es el siguientes: "Observar el comportamiento documental de la producción científica respecto al tema de estudio".

La metodología propuesta para alcanzar estos objetivos ha sido la siguiente, para lograr el objetivo general, hemos realizado una revisión bibliográfica en dos importantes bases de datos en Ciencias de la Salud, una de ellas representa a la comunidad científica Internacional y se corresponde con la base de datos Medline, y la otra base de datos representa a la comunidad científica Nacional, y se corresponde con la Base de datos Medline e Índice Médico Español (IME). Una vez obtenida ambas consultas bibliográficas, hemos recuperado cada uno de los documentos primarios, es decir, hemos recuperado todos los artículos propuestos por la bases de datos.

Las estrategias de búsqueda bibliográfica $o$ matriz de búsqueda se ha realizado de la siguiente manera:

Sólo nos interesaban para nuestra investigación, los artículos que estudiaran o investigaran sobre inmigración y salud, por este motivo para acotar la búsqueda hemos utilizado los siguientes descriptores de ciencias de la Salud o términos Mesh a través del tesauro:
- Emigración e inmigración (introducido en medline con las palabras: Emigration and Immigration).

- Salud (introducido en medline con la palabra: Health ).

- Servicios de salud (introducido en medline con las palabras: Health services).

- Accesibilidad a los servicios de salud (introducido en medline con las palabras: Health Services Accessibility ).

Por otra parte, para garantizar que la bibliografía fuera exclusivamente española, se limito la búsqueda por el idioma en la que estaba escrito el artículo, seleccionando sólo el lenguaje (Language o LA) español y limitando el país de publicación ( Country of Publication o CP) a solamente España. Finalmente, el último filtro que utilizamos fue la limitación temporal, abarcando el periodo de estudio desde 1980 hasta el 2001 (Publication Year o $\mathrm{PY})$.

Una vez recuperados los artículos, a través del Catálogo colectivo de las publicaciones periódicas de bibliotecas españolas en Ciencias de la Salud C17, se procedió a clasificar los artículos, analizando los discursos dominantes en cada uno de ellos, utilizando el método lógico, mediante agrupación o captación del fondo y el sentido temático o ideológico,, clasificando posteriormente las ideas. Posteriormente, para alcanzar el objetivo secundario propuesto, la metodología utilizada a sido realizando un estudio bibliometrico o de análisis del comportamiento documental. Para ello, se han estudiado básicamente los campos CP, PY de los registros o artículos.

\section{RESULTADOS}

El total de publicaciones registradas con los filtros propuestos anteriormente han sido de:

En la base de datos Medline 72 artículos.

En la base de datos IME 8 artículos, los cuales también se encontraban indizados o formaban parte en la base de datos Medline.

\section{Datos iniciales:}

De el total de las 72 publicaciones encontradas, nosotros hemos analizado 45 , debido a que un $22 \%$ se repetían en distintos años de publicación, 
llegando a las mismas conclusiones y tan sólo han aportando como novedad más sujetos en el estudio.

Las pérdidas de datos o documentación se sitúan en torno a un 5,5\%, lo que equivale a 4 artículos que fue imposible su recuperación para el posterior análisis.

Un $10 \%$ del total de artículos, fueron excluidos del estudio debido a que no abordaban como tema principal, la inmigración y salud, sino que entre la muestra que utilizaban estos artículos se encontraban fortuitamente población inmigrante pero no tenían como objeto principal de estudio la inmigración y la salud.

Respecto al comportamiento documental podemos afirmar que la producción científica a lo largo del periodo de estudio 1980 - 2001, no se ha mantenido estable, sino que presenta una tendencia alcista con dos grandes picos de producción producidos en el año 1995 y 2000, con un porcentaje del $11,11 \%$ y $24,44 \%$ respectivamente, cabe destacar que en el año 2001, la producción de artículos sobre inmigración se divide a la mitad, o sea al $11,11 \%$, respecto al año anterior, posiblemente por que no estén indizadas el total de las publicaciones que pertenecen al año 2001, y por lo tanto no se ha podido recuperar todo el año para su estudio (Fig. $1)$.

Como se puede observar en la figura 2, las tres comunidades españolas más frecuentes donde se han realizado estudios de inmigración y salud, ha sido Cataluña con un $42,1 \%$, seguida muy de cerca por la Comunidad de Madrid con un 31,57\% y lejanamente le sigue Canarias con un 10,52\%.

Respecto a la procedencia de los inmigrantes que formaban parte de esos estudios, más de la mitad, concretamente el $57 \%$ proceden de África, el resto se reparte entre; por un lado el $30 \%$ de inmigración Latinoamericana y el 13\% de inmigración procedente de Asia

En cuanto al objetivo principal, es decir, intentar conocer la imagen de una posible clasificación de los textos, mediante el análisis del discurso dominante del artículo, tenemos que señalar que de los 45 artículos analizados podemos decir que existen dos discursos dominantes, que se presentan a continuación:

"La inmigración produce un aumento de las tasas de Enfermedades Infecciosas y Tropicales".
"Utilización de los Servicios Sanitarios por los inmigrantes ".

A continuación abordamos cada uno de estos discursos:

\section{1. " La inmigración produce un aumento de} las tasas de Enfermedades Tropicales e Infecciosas ".

El primer grupo de enfermedades dominantes en los discursos de la bibliografía referida son las enfermedades tropicales, más concretamente las patologías cuya causa u origen se debe a un parásito. La enfermedad estrella en este sentido, ha sido la Neurocisticercosis, seguido más lejanamente de la malaria o paludismo, esquistosomiasis, etc.

El estilo literario que se da a los artículos que tratan estas enfermedades, enfatizan en el aspecto de que son enfermedades importadas, ajenas a nuestras enfermedades, esto se puede apreciar observando simplemente los títulos de algunas de las publicaciones, que mostramos a continuación:

"¿Neurocisticercosis: una enfermedad importada?".

Medicina clínica. 2001 Feb 24; 116(7): 261-3.

"Enfermedades tropicales importadas: experiencia de una unidad especializada en un hospital general."

Revista clínica española. 2000 Oct; 200(10): 533-7.

"Neurocisticercosis en España. Apropósito de 4 casos vistos en pacientes inmigrantes de países endémicos ".

Anales de medicina interna.1999 Feb; 16(2): 89-91.

"Consideraciones epidemiológicas en enfermedades infecciosas inesperadas. Problemas de migraciones "

Anales de la Real Academia Nacional de Medicina.1997; 114(1): 81-99; discusión 99-101.

"Malaria importada por los inmigrantes en Cataluña".

Medicina clínica. 1995. 21; 104(2): 45-8.

"Paracoccidioidomicosis importado. A propósito de 2 casos " 
Medicina clínica.1995. Dic 2; 105(19): 756.

En el segundo grupo las enfermedades infecciosas las más nombradas son, principalmente la TBC y con muchas menos alusiones el VIH. En estos artículos muestran en sus conclusiones, una clara relación estadística de como la inmigración a aumentado las tasas de enfermedades infecciosas.

A continuación se muestran unos párrafos que pone de manifiesto esta relación de aumento de las tasas de TBC y aumento de la inmigración.

"Prevalencia de la infección tuberculosa en los inmigrantes magrebies"

Medicina clínica. 2000 Feb 26; 114(7): 245-9

ANTECEDENTES: Los inmigrantes magrebíes son un grupo de riesgo elevado para la tuberculosis. Las características especiales de este grupo (....., dificultades de la comunicación, estado de ilegal) pueden tener una influencia negativa en tuberculosis....

MATERIAL Y MÉTODOS: Un estudio descriptivo.... Los inmigrantes magrebíes mayores de 14 años atendidos en el Centro de Salud ColladoVillalba, Madrid, España $(\mathrm{n}=520)$..

CONCLUSIONES: "El predominio de la infección de la tuberculosis de inmigrantes magrebíes residentes en España es muy alto. Las condiciones vivas particulares de este grupo pueden tener una influencia negativa ".

"Tuberculosis en inmigrantes recientes en Barcelona"..

Medicina clínica. 1996 Apr 13; 106(14): 525-8.

MATERIAL Y MÉTODOS. "los porcentajes más altos de positividad fueron en pacientes de Subsaharian África (el 52\%) seguida por el este europeo y asiáticos (el 44\%), suramericanos y centroamericanos (el 38\%) y medio-este y África norteña (el 34\%)".

CONCLUSIONES: "la prevalencia de la infección tuberculosa y de la enfermedad tuberculosa es alto en inmigrantes de Barcelona, España".
Respecto a los artículos que versan sobre el VIH y la inmigración, hay que señalar que son muy escasos, y que siempre destacan que no hay una clara relación estadística entre la llegada de inmigración y un aumento en la prevalencia del VIH en España, es más se afirma que no es común la administración de drogas por vía parenteral entre la población inmigrante y este aspecto puede haber influido en el estudio.

"Enfermedades infecciosas y características sociodemográficas del los inmigrantes extranjeros en la penitenciaría central para los hombres en Barcelona".

Revista española de salud pública. 1998 MayJun; 72(3): 197-208.

CONCLUSIONES: Los presos nativos tenían $\mathrm{VIH}$ en un mayor grado o carga de VIH que los inmigrantes, el uso de drogas inyectadas, es más común entre los naturales o nativos ,.......

\section{"Características diferenciales del SIDA en inmigrantes extranjeros ".}

Gaceta sanitaria. 2000 May-Jun; 14(3): 189-94

CONCLUSIONES: El porcentaje de inmigrantes con SIDA en Barcelona es bajo. Los inmigrantes son con frecuencia hombres, que menudo viven en el centro o interior urbano y se inyectan con menos frecuencia que los usuarios de droga nacionales o autóctonos.

A continuación presentamos más detalladamente el segundo discurso:

\section{2. "Utilización de los servicios de sanitarios"}

Por una parte la bibliografía hace referencia a la preocupación de los problemas pediátricos que los hijos de los inmigrantes pudieran tener en España. En concreto se afirma que España es el país con más posibilidades de inmigración de África, de Latino-América y de Filipinas, así pues es absolutamente necesario que los pediatras españoles sepan de las enfermedades pediátricas de estos grupos y formas para su integración. Esta preocupación sobre los conocimientos médicos que se deben tener, también se hace extensible a las enfermedades tropicales. 
También queda reflejado en la bibliografía, que existe preocupación respecto a la situación de ilegalidad que conduce a pésimas condiciones sanitarias y de la barreras lingüísticas, que empeoran la situación. Ya desde el año 1994 y 1995 encontramos un artículo para cada año respectivamente, que se preocupa de esta cuestión y propone alternativas para paliarlo, como la puesta en marcha de programas interdisciplinarios socio-sanitarios que ayuden a superar las barreras culturales e idiomáticas. Ha pesar de que la primera advertencia de estas barreras se hizo en el 1994 y seguidamente en el año 1995, hasta el año 1999 no se encuentra ningún registro que retome esta preocupación. Después del año 1999 no se recuperan más registros que aborden esta cuestión.

Respecto a lo que propiamente se trata de la utilización de los servicios sanitarios, debemos destacar que el INSALUD es el abastecedor principal de los servicios médicos a los inmigrantes, y especialmente los servicios de urgencias.

Los principales motivos de consulta que hemos encontrado según la bibliografía en los pacientes inmigrantes, en general fueron los siguientes:

\section{$1^{\mathrm{a}}$. Dolores musculares.}

$2^{\mathrm{a}}$ Dolencias respiratorias localizadas en el tracto superior.

$3^{\text {a }}$ Accidentes.

También existen referencias, aunque son raquíticas, que señalan los problemas en la salud mental que pueden tener los inmigrantes.

\section{DISCUSIÓN Y CONCLUSIONES}

Es evidente que el total de la bibliografía existente en España es escasa, teniendo en cuenta que las consultas han sido realizadas por un lado en la base principal de Ciencias de la Salud a nivel Internacional que es Medline y por otra parte en la única base de datos médica española que es el Indice Médico Español. Nosotros creemos que existe más documentación al respecto, pero que no ha podido ser recuperada por tratarse de publicaciones a nivel interno de distintas Instituciones sanitarias y sociales públicas u ONGs que trabajen con inmigrantes. Aún así, esta cifra de artículos es muy superior a los encontrados en revistas de enfermería, lo que nos puede dar una idea del grado de implicación de los diferentes colectivos profesionales (Garvi M. 2003).

Respecto a las localizaciones más frecuentes donde se han realizados los estudios de inmigración y salud, no nos sorprende que hayan sido Cataluña y la Comunidad de Madrid, seguido de la Comunidad Canaria, puesto que estas localizaciones son donde se producen más asentamientos de la población inmigrante por ese motivo, nos parece normal que si realmente son las comunidades con más población inmigrante dentro de España, realicen más estudios sobre inmigración que el resto de las comunidades autónomas. Pero el que exista más población no significa en estas comunidades, no nos debe parecer que se esté produciendo "una" invasión en ambas comunidades, puesto que España es el país con menor número de inmigrantes en comparación con otros países europeos, en concreto el porcentaje que representa España es del 1,6\% frente a nuestros vecinos franceses que representan el 8,2\% o Bélgica que está a la cabeza con el $10 \%$ de inmigrantes en relación a la población global (Calvo T. 2000).

Cabe destacar a este punto, que los inmigrantes europeos según el boletín estadístico de octubre de 1999 del INSERSO son 329.956 , es decir el 45,8\% del total de los inmigrantes, y sin embargo en la literatura médica española no hay constancia de ellos, frente a los latino-americanos y los africanos que disputan el título o la autoría de aumentar las tasas de enfermedades tropicales e infecciosas. Esta reflexión nos podría llevar a abrir otra nueva línea de investigación, y es ¿a qué llamamos inmigrantes los profesionales de la salud?, ¿qué características tienen estos pacientes para que sean considerados inmigrantes?, deben ser de color diferente, deben tener antecedentes de drogadicción, deben vivir en condiciones de precariedad y hacinamiento, etc.. para considerarlos inmigrantes ó ¿pueden tener una posición socioeconómica alta para que aún así los consideraríamos inmigrantes?, es decir, ¿existen inmigrantes de primera y de segunda categoría? ó todos los inmigrantes son iguales. Debemos reflexionar si tenemos verdaderamente el mismo concepto de un paciente centro-europeo con una posición acomoda que viene a España por motivos laborales o de 


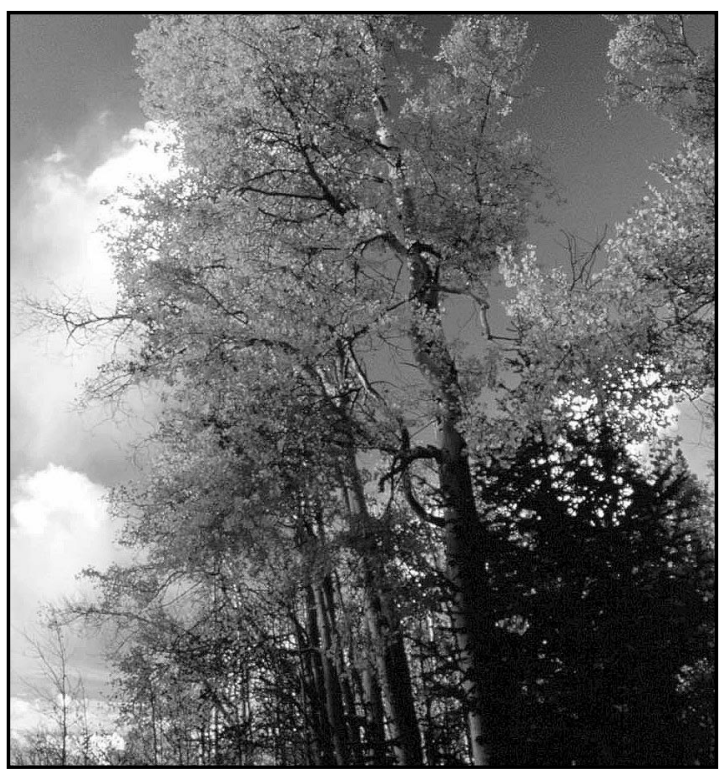

vacaciones y durante su estancia permanece ingresado o en urgencias, que de un paciente que llega al hospital con hipotermia porque acaba de llegar en una patera. Aunque las respuestas a todas estas preguntas requiere un estudio mucho más profundo para poder contestarlas, creo que podíamos anticipadamente responder, que no tenemos la misma conceptualización del termino inmigrante dependiendo de factores económicos, aspecto general, procedencia, etc , pero evidentemente, esto no conlleva a suponer que el personal de enfermería cuide de manera diferente a las diferentes clasificaciones o tipologías de inmigrantes que haya elaborado.

Por otra parte, también hay que tener en cuenta que en los estudios en los que el resultado es un aumento de las tasas de las enfermedades tropicales, incluyen no sólo pacientes inmigrantes sino también pacientes nativos que han realizado viajes a zonas endémicas y cooperantes. Por lo tanto habría que valorar a la hora de prevenir este tipo de enfermedades no sólo a la población inmigrante que proviene de esas zonas sino también a los viajeros nativos que viajan a esas zonas, por lo que el problema no está en el agente transmisor si es español, latino-americano o magrebí sino en sus prácticas higiénico-sanitarias, principalmente el lavado de manos después de defecar y antes de las comidas y beber agua embotellada, porque a las enfermedades que estamos refiriéndonos son debi- das principalmente a parásitos intestinales que se albergan en el hombre, se eliminan por las heces y pasan nuevamente al hombre por contacto fecaloral. Esto no significa que se deba restar importancia a las enfermedades infeccionas "importadas" por los pacientes inmigrantes, sino que se requiere un estudio profundo de las características demográficas de estos pacientes, para poder estudiar posibles soluciones, A este respecto, cabe destacar que son escasos o con muestras pequeñas los estudios que pongan de manifiesto las características sociodemográficas, uno de estos estudios con una muestra más o menos representativa es el estudio realizado por Sanz. B, Torres. A.M y Schumacher (2000), en la CAM a 300 inmigrantes elegidos aleatoriamente, durante el año 2000. Podemos destacar algunas de esas características que son relevantes en materia de salud:

- La edad media fue de 32,6 años (CI del 95\%, 31,6-33,7). Con 6,6 años que vivían en España.

- El 30,9\% (CI 24,3-35,9) vivían en situación de irregularidad administrativa y de cobertura de salud.

- Una de cada cinco personas no tenía ni agua corriente ni recogida de desperdicios.

Para establecer un programa de salud es imprescindible establecer el diagnostico de salud de la comunidad, y para ello debemos conocer a la población, sus características demográficas, conocer las condiciones de vida y los problemas asociados a estas condiciones; infravivencia, no tener trabajo, falta de escolarización, etc....Identificar sus necesidades y problemas de salud, saber como afrontan la enfermedad, estudiar los acceso y uso de los servicios de salud, etc de toda la población que constituye esa comunidades, es decir, tanto de la población inmigrante como no inmigrante . En este sentido un ejemplo son las diferencias que existen en los calendarios de vacunación entre la población autóctona y la población inmigrante, los resultados más relevantes los aportan estos de dos estudios que recogemos a continuación:

El primero realizado en Madrid a 76 inmigrantes de mayoritariamente de origen Latinoamerica-no (el $70 \%$ ), en dicho estudio se concluía que la presencia de hepatitis B era baja en esta población (Lacalle M. 
2000) . Sin embargo, el segundo estudio con 1226 inmigrantes se centra en la situación inmunológica de la hepatitis B en inmigrantes y las estrategias de vacunación. Sus resultados muestran que los inmigrantes procedentes de África Sub-Shariana presentaban un $29,1 \%$ de adecuada vacunación de la hepatitis $\mathrm{B}$, frente a los inmigrante que procedían de América que presentaban una tasa de vacunación del virus de la hepatitis B mucho más superior (el $91,5 \%)$, estas tasas deben tomarse con precaución porque variaban con la edad de los inmigrantes. Concluye el estudio, diciendo que la edad y el lugar de nacimiento explican la conveniente inmunización de la hepatitis B (Romea S. 1997), y por lo tanto al elaborar el programa de salud de la comunidad y en especial la campaña de vacunación se deben tener en cuenta estas diferencias.

Otra imagen o estereotipo que existe sobre la salud de los inmigrantes, es que estos llegan o tiene un estado nutricional deficiente. A este respecto podemos afirmar que a través de la bibliografía investigada, sólo existe un estudio exhaustivo, que realice una evaluación de la nutrición en los inmigrantes, y en este caso de los inmigrantes de origen magrebí. Este estudio concluye que un bajo porcentaje de pacientes muestra una suave desnutrición aguda, relacionada probablemente la privación del alimento y los hábitos dietéticos pobres experimentados durante el proceso de la inmigración. La desnutrición crónica no fue observada en este estudio, en el momento inmediato de la llegada a otro país, esto no significa que los inmigrantes posteriormente puedan llegar a tener un estado nutricional deficiente.

Respecto a los motivos de consulta o la utilización de los servicios sanitarios hay que señalar que algunos estudios excluyen en su investigación las causas obstétricas, que suponemos pueden ser el mayor motivo de hospitalización y consultas pediátricas en Atención Primaria.

Gracias a la lectura de los artículos, debemos romper algunos estereotipos respecto al estado de salud de la población inmigrante. La mayoría de los inmigrantes que llegan a nuestro país son personas jóvenes y sanas que muchas veces enferman aquí por la situación de pobreza y exclusión en la que se encuentran. Son por lo tanto las situaciones de marginación, soledad, hacinamiento y pobreza con las que se enfrentan durante el proceso migratorio las que contribuyen a que presenten determinadas enfermedades y especialmente las de causa infecciosa (3).

Aunque en la bibliografía se escribe sobre la necesidad de programas interdisciplinarios sociosanitarios que ayuden a superar las barreras culturales e idiomáticas, no se deja reflejado cómo deberían ser estos programas, si existen experiencias en otros países, etc . Personalmente pensamos que sería necesario crear un " mediador sanitario -cultural ", cuya principal función sería orientar al personal sanitario en las relaciones interculturales. El mediador sería una persona del grupo de inmigrantes que conoce bien la cultura y la lengua. Este hace de interlocutor, facilita la comunicación y ayuda al personal médico a establecer el diagnóstico médico, y al personal de enfermería a establecer sus diagnósticos de enfermería y cuidados, además ayuda al enfermo a entender el tratamiento o pruebas complementarias necesarias.

También observamos, la necesidad de la puesta en marcha de programas de formación destinados al personal sanitario, especialmente al personal de enfermería por ser el colectivo que más tiempo pasa junto al paciente, en temas de salud de diversidad cultural, que le ayuden a superar las barreras culturales.

Respecto al estado de salud de los inmigrantes, las enfermedades mentales, básicamente son la depresión y la ansiedad, que se producen por la situación de desarraigo, choque cultural, ruptura familiar en algunos casos, falsas expectativas, etc.. Pensamos que estos trastornos mentales se podrían prevenir, advirtiendo desde las instituciones el precio personal que tiene que pagar el inmigrante. La inmigración supone un cambio importante en la vida de las personas. De hecho se la considera un trasplante cultural, sobre todo si las diferencias culturales son grandes, y también un segundo nacimiento, una segunda oportunidad (Roca N. 2001). Es evidente que donde primero se sufre la inmigración es en el propio cuerpo: estrés, soledad, disminución de la autoestima, angustia, falta de soporte afectivo, sentimientos de culpabilidad, precariedad, etc. La migración, por si sola, debe ser considerada como un factor de riesgo para la salud psíquica y espiritual (Roca N. 2001). 
Finalmente, como última reflexión; debido a que el proceso migratorio es un proceso cambiante y por lo tanto los discursos que se emiten en las revistas de ciencias de la salud también cambian, los autores de este trabajo, nos hemos planteado una nueva pregunta de investigación, ¿sigue prevaleciendo este discurso o ha cambiado radicalmente en estos últimos cuatro años?. Seguramente no, pero todavía no podemos negarlo con rotundidad ya que el equipo investigador no ha finalizado esta segunda línea de investigación.

\section{BIBLIOGRAFÍA}

- Calvo T. (2000) Inmigración y racismo. Así se sienten los jóvenes del siglo XX. Cauce editorial. Madrid, pag 124-127.

- Carta Magna o Carta Constitucional (1946). Organización Mundial de la Salud (OMS). Disponible el original en ingles en http://whqlibdoc.who.int/hist/official_records/constitution.pdf (última consulta 03-03-2004).
- Garvi M., García S., Villa J.M. (2003) La población inmigrantes desde la perspectiva asistencial. Enfermería Clínica Núm. 256-257. Julio-agosto. pag: 26-30.

- Lacalle-Rodriguez,M., Gil G., Sagardui J.K., González E., Martínez R., Orden B. (2000) Resultados de la aplicación de un examen de salud en población inmigrante. Atención Primaria. May 31; 25(9): 634-8.

- Romea S., Duran E., Cabezos J., Bada J.L.(1997) Situación inmunológica de la hepatitis B en inmigrantes. Estrategias de acción. Medicina Clínica (Barcelona). Nov 15; 109(17): 65660.

- Roca i Capará, N.(2001) Inmigración y salud (I). Situación en España. Rol de Enfermería ; 24(10):682-686.

- Roca i Capará, N. (2001) Inmigración y salud (II). La salud de las personas inmigradas. Rol de Enfermería ; 24(11):792-798.

- Sanz,-B; Torres,-A-M; Schumacher,-R. (2000) Características sociodemográfica y utilización de servicios sanitarios por la población inmigrante residente en un área de la Comunidad de Madrid. Atención Primaria. Sep 30; 26(5): 314-8.

- WHO, Primary Health Care: Alma-Ata 1978 (Ginebra: Organización Mundial de la Salud, 1979), 79 artículo X.

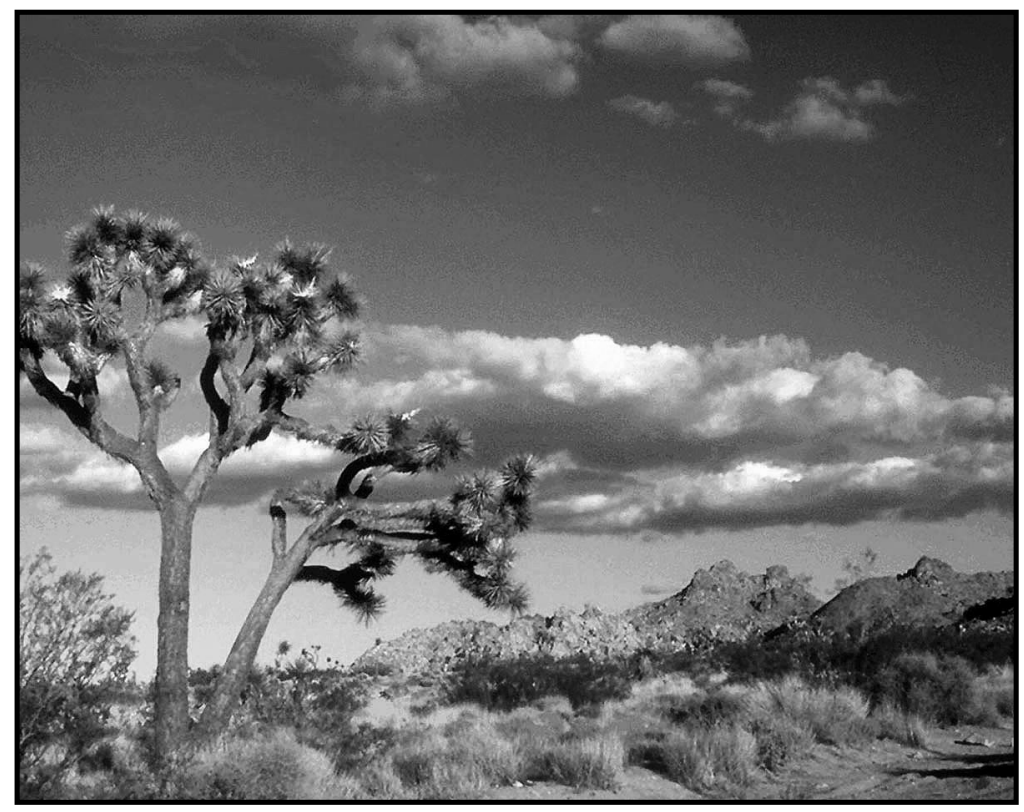

